\title{
New Surgical Technique: Dorsal Osteosynthesis Approach for Tibial Defect Reconstruction
}

\author{
Dzintars Ozols, $\mathrm{MD}^{1,2}$ Karlis Verdins, $\mathrm{MD}^{1}$ Janis Zarins, $\mathrm{MD}^{1,2}$ \\ ${ }^{1}$ Department of Hand and Plastic Surgery, Microsurgery Centre of \\ Latvia, Riga East University Hospital, Riga, Latvia \\ ${ }^{2}$ Riga Stradiṇš University, Riga, Latvia \\ Address for correspondence Dzintars Ozols, MD, Microsurgery Centre \\ of Latvia, Hipokrata 2, Riga, Latvia, LV-1038 \\ (e-mail: dz.ozols@gmail.com).
}

J Reconstr Microsurg Open 2016;1:48-52.

The new dorsal osteosynthesis (reconstruction) approach was developed to reduce the risks of plate exposure, infection, and early removal in difficult cases such as Gustilo-Anderson type IIIB or C open tibial fractures and Cierny-Mader type III and IV chronic osteomyelitis. The advantages of this method are that the reconstruction with microvascular or pedicle fibula flaps and plating can be done through healthy noncompromised posterior compartment-no separate incision for osteosynthesis or flap insertion is needed.

\section{Material}

Retrospective cohort study was performed to evaluate the functional outcome, union rate, and complications after tibia reconstruction. Eleven patients underwent dorsal osteosynthesis for tibia defect reconstruction with free or pedicle fibula flaps in the Microsurgery Centre of Latvia between 2010 and 2015. All patients had a history of open tibia fractures. Patients were divided in three groups. In the first group, three patients developed late complications after previously done bone and soft tissue reconstructions. The second group included six patients with chronic osteomyelitis after osteosynthesis with a plate, using classical anteromedial or anterolateral approaches without any reconstruction. The third group included two patients with Gustilo-Anderson type IIIB and IIIC tibia fractures treated with monolateral and Ilizarov apparatus, also without any reconstruction. Group characteristic are shown in -Table 1.

\section{Surgical Procedure}

Patient is operated in the abdominal position. Posterior compartments of both legs are on top, and feet are slightly flexed.

In cases of free flap incision going from medial side through posterior compartments, gastrocnemius muscles are retracted laterally or medially. Soleus muscle has to be

received

July 16,2015

accepted after revision

September 27, 2015

published online

January 21, 2016 dissected and sharply detached from tibia. Posterior tibial artery (PTA) and tibial nerve (TN) can be observed till bifurcation. Tibialis posterior, flexor hallucis longus, and flexor digitorum longus muscles are gently detached from medial side to avoid damaging of motor nerve branches. After dividing last muscles, dorsal side of the tibia can be fully observed. Posterior tibial vessels are dissected starting from peroneal artery (PA) till medial ankle and can be used at any level for microvascular end-to-side anastomosis. Contralateral fibula flap can be harvested from dorsal side of the leg.

If pedicled fibula flap is used for reconstruction, skin incision can be done from lateral side, closer to fibula, for better exposure of PA. ${ }^{1-3}$ Flaps can be proximally (anterograde flow) or distally (retrograde flow) based depending on defect localization. ${ }^{4}$ Proximally based pedicle vascularized fibula graft can be used for tibial shaft defects and retrograde flap is recommended for distal tibial defects. It is not necessary to dissect PTA and TN (-Figs. 1 and $\mathbf{2}$ ).

After flap harvest, osteosynthesis is done through dorsal approach-plate is mounted from dorsal side of the tibia. Some additional cortical screws can be used to fix a single or double barrel. Some difficulties can be anticipated in inserting the upper part of plate as bifurcation of PA and PTA lies there. Gentle distraction has to be applied to pull away blood vessels and insert screws. The skin paddle has to be pushed from the posterior side to the anterior side. After the dorsal side closure, the leg is flexed in knee joint and skin paddle is fixed. Some defects can be left for secondary healing.

We recommend leaving the external fixator on till skin heals, and remove it in outpatient clinic after 4 to 8 weeks when circular cast can be safely applied.

\section{Methods for Evaluation}

Patient's functionality was observed by Lower Extremity Functional Scale (LEFS) score. ${ }^{5} \mathrm{X}$-rays, sensation, range of
Copyright $\odot 2016$ by Thieme Medical Publishers, Inc., 333 Seventh Avenue, New York, NY 10001, USA. Tel: +1(212) 584-4662.

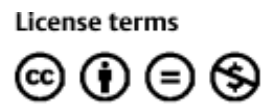

$10.1055 / \mathrm{s}-0035-1570532$ ISSN 2377-0813. 
Table 1 Group characteristics

\begin{tabular}{|c|c|c|c|c|c|}
\hline & Age & Gender & Problem & Bone defect & Reconstruction \\
\hline \multicolumn{6}{|c|}{ First group } \\
\hline 1. & 26 & M & Nonunion & Tibial shaft, $2 \mathrm{~cm}$ & Distal pedicle, $7.9 \mathrm{~cm}$ \\
\hline 2. & 43 & $\mathrm{M}$ & Nonunion & Tibial shaft, $14 \mathrm{~cm}$ & Proximal pedicle, $20.2 \mathrm{~cm}$ \\
\hline 3. & 45 & M & Osteomyelitis 3A & Tibial shaft, $14 \mathrm{~cm}$ & Contralateral single barrel, $18 \mathrm{~cm}$ \\
\hline \multicolumn{6}{|c|}{ Second group } \\
\hline 4. & 54 & M & Osteomyelitis 4A & Distal tibia, $13 \mathrm{~cm}$ & Contralateral single barrel, $19 \mathrm{~cm}$ \\
\hline 5. & 54 & $\mathrm{~F}$ & Osteomyelitis $3 \mathrm{~A}$ & Distal tibia, $3.5 \mathrm{~cm}$ & Distal pedicle, $8.5 \mathrm{~cm}$ \\
\hline 6. & 57 & $\mathrm{M}$ & Osteomyelitis 4A & Distal tibia, $9.3 \mathrm{~cm}$ & Contralateral double barrel, $12 / 10 \mathrm{~cm}$ \\
\hline 7. & 41 & $\mathrm{M}$ & Osteomyelitis $3 \mathrm{~A}$ & Distal tibia, $10.7 \mathrm{~cm}$ & Distal pedicle, $15,4 \mathrm{~cm}$ \\
\hline 8. & 54 & M & Osteomyelitis 4C & Tibial shaft, $4.6 \mathrm{~cm}$ & Proximal pedicle, $9,6 \mathrm{~cm}$ \\
\hline 9. & 40 & M & Osteomyelitis 3A & Distal tibia, $4.8 \mathrm{~cm}$ & Distal pedicle, $10.2 \mathrm{~cm}$ \\
\hline \multicolumn{6}{|c|}{ Third group } \\
\hline 10. & 24 & $\mathrm{M}$ & Gustilo-Anderson IIIB & Tibial shaft, $6.5 \mathrm{~cm}$ & Contralateral double barrel, $9.6 / 8 \mathrm{~cm}$ \\
\hline 11. & 25 & $\mathrm{M}$ & Gustilo-Anderson IIIC & Tibial shaft, $9.3 \mathrm{~cm}$ & Contralateral double barrel, $11.7 / 11.6 \mathrm{~cm}$ \\
\hline
\end{tabular}

motion, leg length, and Medical Research Council (MRC) scale were evaluated.

\section{Results}

Seven patients responded for the study. Mean follow-up time for five patients was 2 years and 8 months; for two patients, it was less than 1 year. Mean LEFS score was 56.3 points (43-69) or $70.35 \%$ (53-86.2\%) from maximal ability which is $100 \%$ according to the scale. Most of the difficulties were associated with high-intensity workouts such as running or jumping. Normal daily activities such as walking and going up or down the stairs can be done without any difficulties. No one had abnormal sensations at the TN innervations zone. The MRC scale score was M5 for all patients. M5 was evaluated if patient were able to stand in the toe position against the gravity of full resistance. Limb length differences were corrected with orthopedic foot wear (-Figs. 3 and 4; - Table 2).

\section{Discussion}

Many surgical approaches are available for tibia osteosynthesis. The main approaches for tibial reconstruction are anteromedial, anterolateral, posteromedial, and posterolateral. Every approach has its anatomical limitations, advantages, and disadvantages. ${ }^{6}$ The anteromedial approach is the most commonly used for distal tibial shaft reconstruction. The main disadvantage is low blood supply to the skin and subcutaneous tissue which can lead to exposed hardware. The anterolateral approach is used when the medial soft tissues are compromised. The exposure is more difficult
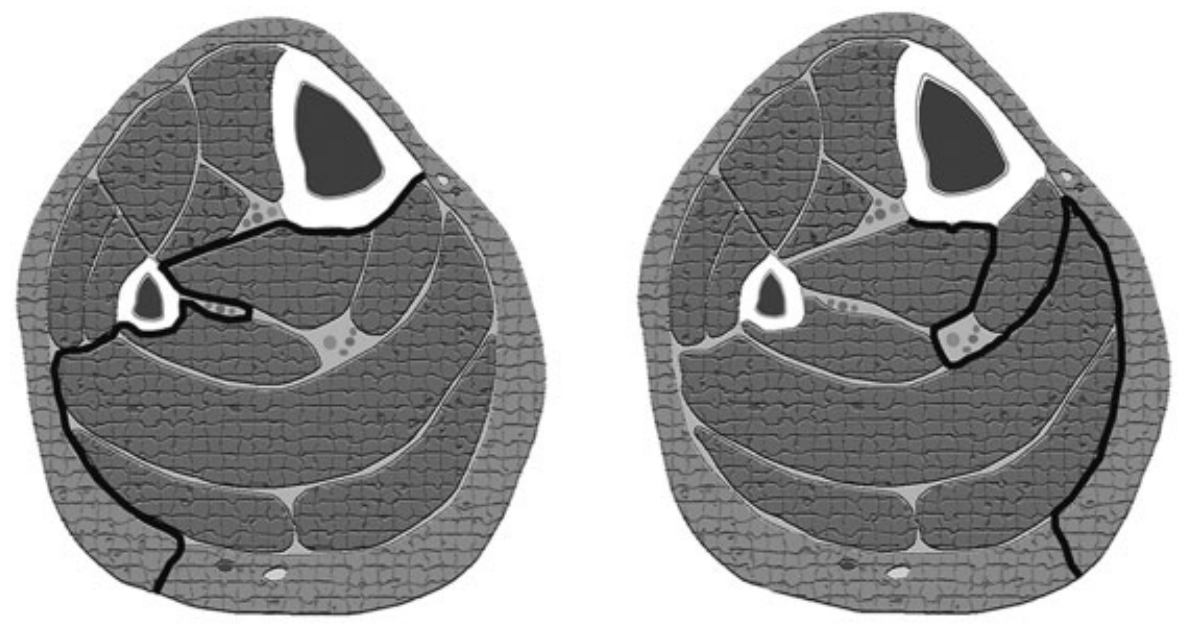

五

Fig. 1 The cross-section differences for the pedicle (left) and free flap (right). 


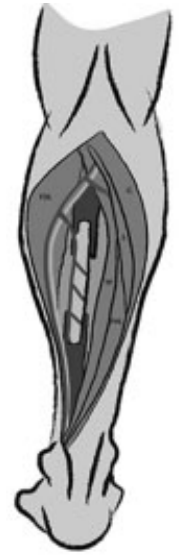

五

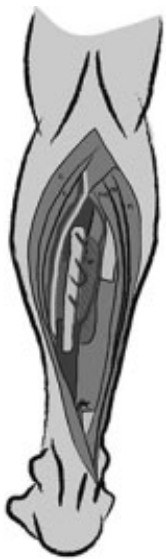

安

Fig. 2 The differences for flap vascular pedicles (free flap on the left and pedicle flap on the right).

because of a risk of damaging anterior tibial artery and deep peroneal nerve. ${ }^{7}$ Classical osteosynthesis methods are developed to avoid exploration and to reduce the risk of damaging lower limb blood vessels or nerves. To reconstruct the tibia with a free or pedicle flaps, blood vessels have to be explored. Using dorsal osteosynthesis approach, a good vascular exploration for microvascular anastomosis or pedicle flaps is achieved.
The osteosynthesis hardware placed under microvascular free flap can be exposed if total or even marginal skin necrosis occurs and reoperations with new flaps might be necessary to close the defect and prevent infection. The dorsal osteosynthesis approach was developed to secure metal plates and reduce a risk of failure. Skin flap's paddle side can be healed by secondary intention without plate exposure. Of 11 patients in our study, 5 were healed by secondary intention. No plate infections were observed. Delayed postoperative complications include nonunion, recurrence or development of osteomyelitis, failure of fixation, fibular fracture, sensory disturbances, contractures, and deformities. ${ }^{8,9}$ Graft fractures are the most common late postoperative complication. Previous authors have found the incidence of fractures between 20 and $40 \% .{ }^{10}$ One patient in our study had a broken plate with bone fracture. Patient specified that too early walking and exercises were applied on the reconstructed leg. The broken plate was removed and reosteosynthesis done.

The dorsal osteosynthesis approach is developed for the tibia reconstruction with pedicle or free bone (contralateral fibula, iliac crest). Treatment with contralateral fibula is selected for the uncomplicated patients. In these cases double-barrel fibula graft is preferred as fast recovery can be expected and patients can start physical activities after cast is removed.
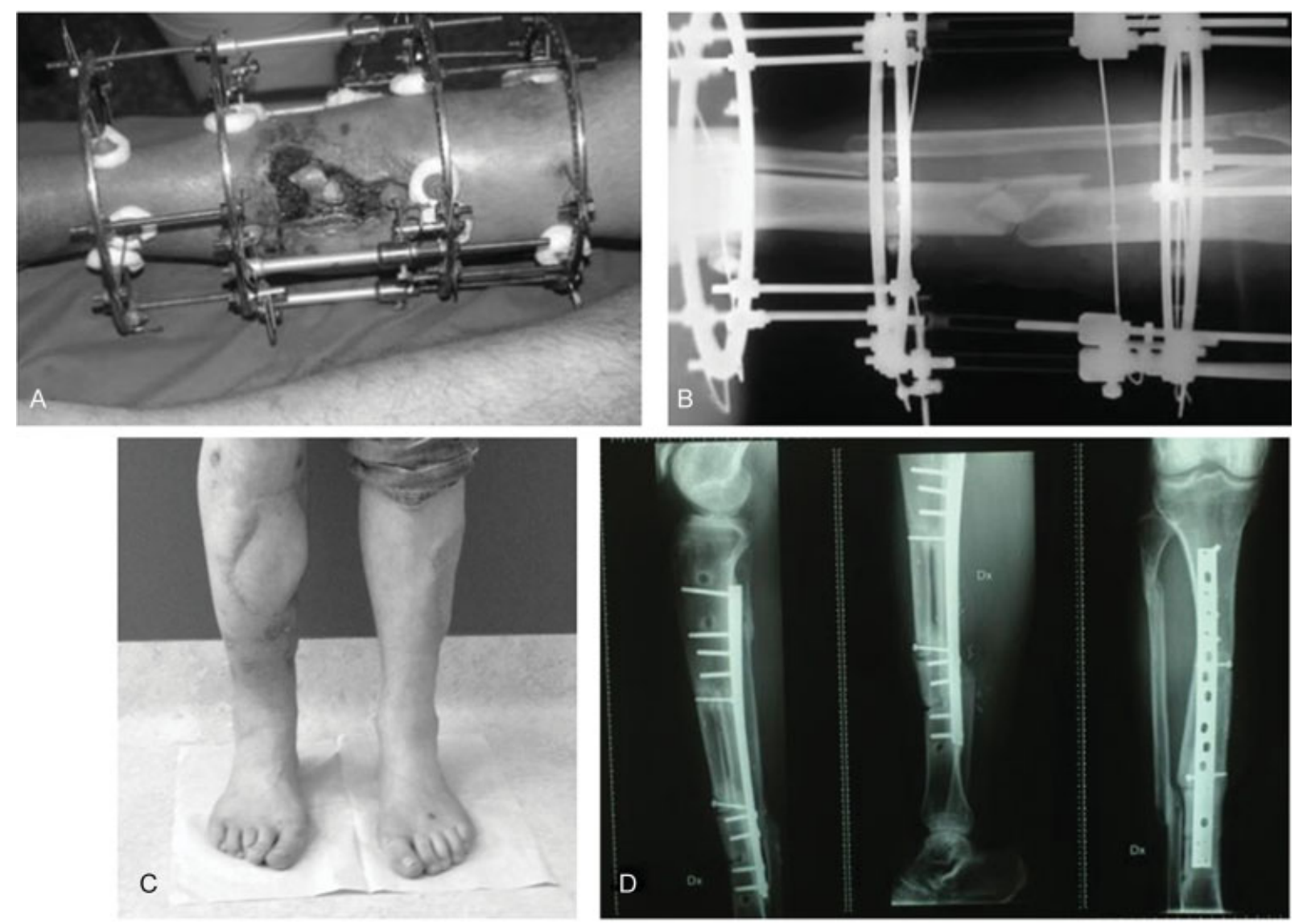

Fig. 3 A 26-year-old patient with open tibial fracture (Gustilo-Anderson type IIIB) treated with llizarov external fixator. (A, B) Infected open tibial fracture. (C, D). Follow-up 2 years after free double-barrel fibula. 

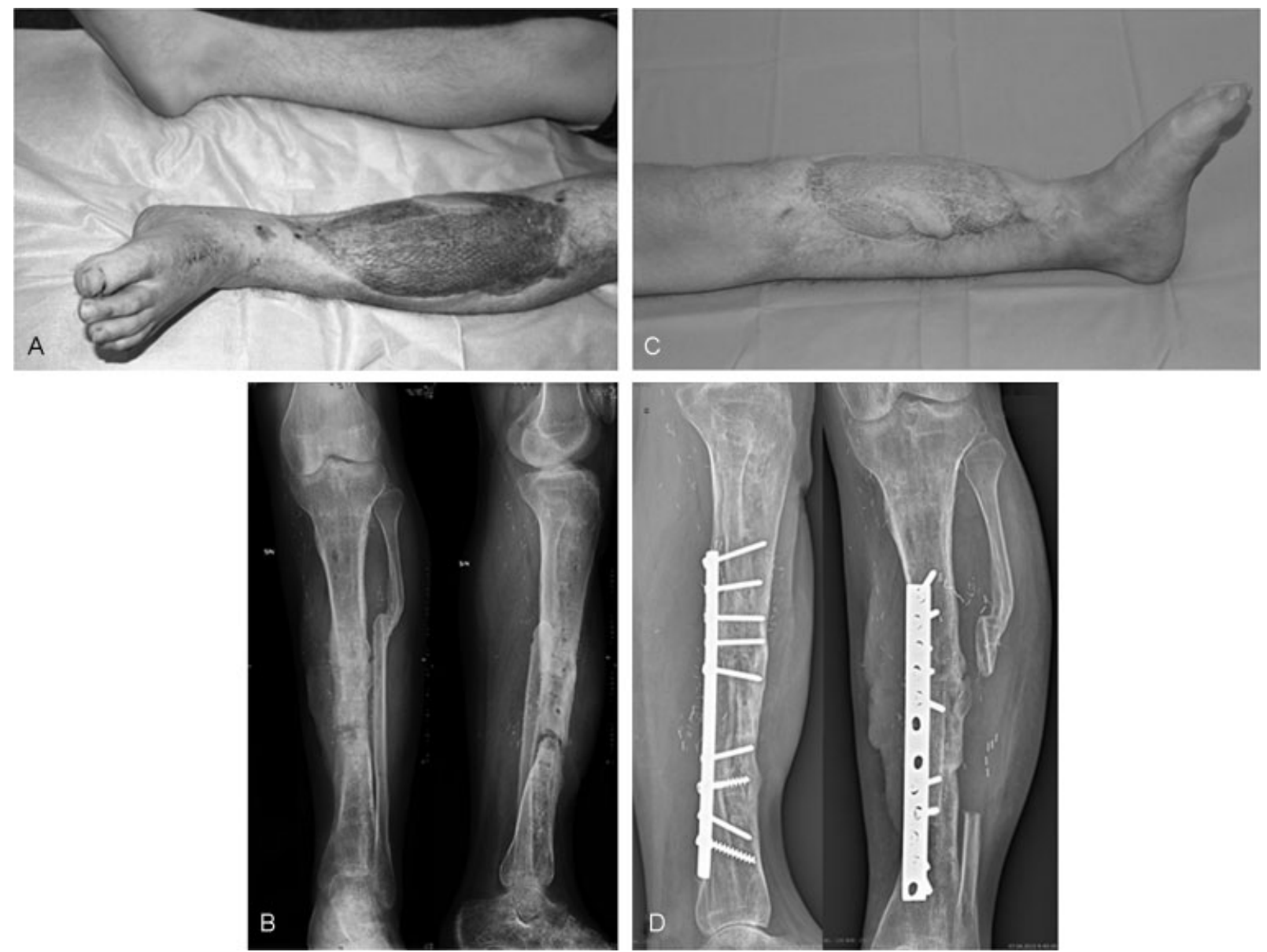

Fig. 4 A 27-years-old patient with left tibial injury (Gustilo-Anderson type IIIC). Free latissimus dorsi, fibula flaps used for early reconstruction. Nonvascularized iliac crest bone used for reosteosynthesis. (A, B) Septic un-union. (C, D) Follow-up 1 year after distal pedicled fibula flap.

Pedicled fibulas are selected for complicated patients as there is higher risk for below-knee amputation. Proximally or distally based pedicle fibula flap can be selected depending on defect localization and vascularity. Fibula fractures are not contraindications for flap selection. The same region operations are preferred for the high failure risk patients as last chance operation before amputation. Salvage of the potential donor side is a huge benefit for these patients as there is not any iatrogenic damage in healthy side.

\section{Conclusion}

The dorsal osteosynthesis approach can be primary choice in cases when large soft tissue defect is expected, which increases the risk of skin paddle necrosis, or as a last reconstruction option if conventional reconstructions fail. Advantages of this method are the following: incision can be made throughout healthy noncompromised tissue; free access for anastomosis; contralateral fibula flap can be harvested from

Table 2 Study outcome data

\begin{tabular}{|l|l|l|l|l|l|l|l|}
\hline Patient & Follow-up & LEFS score & ROM knee & ROM ankle & MRC & Limb, cm & Late complications \\
\hline 1 & 2 y $11 \mathrm{mo}$ & $43,53.75 \%$ & $10-0-130$ & $15-0-50$ & M5 & $82 / 83$ & None \\
\hline 2 & 3 y $1 \mathrm{mo}$ & $55,68.75 \%$ & $0-0-75$ & $20-0-40$ & M5 & $84 / 85$ & None \\
\hline 3 & 3 y 0 mo & $65,81.25 \%$ & $10-0-125$ & $15-0-40$ & M5 & $89 / 92$ & Broken plate \\
\hline 4 & 2 y 9 mo & $44,55 \%$ & $5-0-115$ & $15-0-50$ & M5 & $105 /-$ & None \\
\hline 5 & 8 mo & $63,78.75 \%$ & $0-0-120$ & $15-0-45$ & M5 & $95 / 96$ & None \\
\hline 6 & 10 mo & $69,86.2 \%$ & $5-0-120$ & $10-0-30$ & M5 & $100 / 105$ & None \\
\hline 7 & 8 mo & $55,68.75 \%$ & $0-0-90$ & $10-0-30$ & M5 & $84 / 85$ & None \\
\hline
\end{tabular}

Abbreviations: LEFS, Lower Extremity Functional Scale; MRC, Medical Research Council scale; ROM, range of motion. 
dorsal side; pedicle vascularized fibula flap can be harvested through the same incision; operation time does not increase; and in cases of partial tissue necrosis it can be left for secondary healing.

In spite of small number of patients in our study, we need to continue our work to evaluate the effectiveness of this approach with other methods.

\section{References}

1 Khira YM, Badawy HA. Pedicled vascularized fibular graft with Ilizarov external fixator for reconstructing a large bone defect of the tibia after tumor resection. J Orthop Traumatol 2013;14(2): 91-100

2 Satoh K, Wong F, Shibui S, et al. Effectiveness of the pedicled vascularized fibula graft to reconstruct tibial defects. Eur J Plast Surg 1991;14:274-279

3 Chen ZW, Chen LE, Zhang GJ, Yu HL. Treatment of tibial defect with vascularized osteocutaneous pedicled transfer of fibula. J Reconstr Microsurg 1986;2(3):199-203, 205
4 Minami A, Itoga H, Suzuki K. Reverse-flow vascularized fibular graft: a new method. Microsurgery 1990;11(4):278-281

5 Binkley JM, Stratford PW, Lott SA, Riddle DL; North American Orthopaedic Rehabilitation Research Network. The Lower Extremity Functional Scale (LEFS): scale development, measurement properties, and clinical application. Phys Ther 1999;79(4):371-383

6 Sheerin DV, Turen $\mathrm{CH}$, Nascone JW. Reconstruction of distal tibia fractures using a posterolateral approach and a blade plate. J Orthop Trauma 2006;20(4):247-252

7 Hong J, Zeng R, Lin D, et al. Posteromedial anatomical plate for the treatment of distal tibial fractures with anterior soft tissue injury. Orthopedics 2011;34(6):161-166

8 Nelson JA, Fischer JP, Haddock NT, et al. Striving for normalcy after lower extremity reconstruction with free tissue: the role of secondary esthetic refinements. J Reconstr Microsurg 2016; 32(2):101-108

9 Fischer JP, Wink JD, Nelson JA, et al. A retrospective review of outcomes and flap selection in free tissue transfers for complex lower extremity reconstruction. J Reconstr Microsurg 2013;29(6): 407-416

10 Minami A, Kimura T, Matsumoto O, Kutsumi K. Fracture through united vascularized bone grafts. J Reconstr Microsurg 1993;9(3): 227-232 